\title{
EMBEDDING ITERATES OF ANALYTIC FUNCTIONS WITH TWO FIXED POINTS INTO CONTINUOUS GROUPS $\left({ }^{1}\right)$
}

\author{
BY \\ SAMUEL KARLIN AND JAMES MCGREGOR
}

1. Introduction. Let $f(s)$ be an analytic function with a fixed point $s_{0}$, i.e., a solution of $f(s)-s=0$, in its domain of regularity. We say that $f(s)$ is embedded in a one parameter group near $s_{0}$ if there is a family $f(s ; t)$ of analytic functions, $-\infty<t<\infty$, for each $t$, regular in a neighborhood (depending on $t$ ) of the common fixed point $s_{0}$, such that

$$
\begin{aligned}
f(s ; t+\tau) & =f(f(s ; t) ; \tau), \quad-\infty<t, \quad \tau<\infty, \\
f(s ; 1) & =f(s) .
\end{aligned}
$$

We call the group continuous if the coefficients $c_{k}(t)$ in the expansion

$$
f(s ; t)=s_{0}+\sum_{k=1}^{\infty} c_{k}(t)\left(s-s_{0}\right)^{k}
$$

are continuous. Similarly, the term continuous one parameter semigroup refers to the case when the parameter range is $0 \leqq t<\infty$.

It has been known since Koenigs (1884) that if $\left|f^{\prime}\left(s_{0}\right)\right|$ is not 0 or 1 , then embedding in a continuous one parameter group near $s_{0}$ is always possible. We outline a method of proof in $\$ 2$. The possibility of such an embedding is important in iterative procedures of analysis, in conformal mapping problems, in the study of Markov branching processes and in other applications.

Consequently the embeddings have been studied by numerous authors, of whom we mention only I. N. Baker [1], E. Jabotinsky [2] and G. Szekeres [4]. When $f^{\prime}\left(s_{0}\right)=1$, the embedding is not always possible. It was shown by Baker that the only meromorphic functions $f(s)$, with a fixed point $s_{0}$ such that $f^{\prime}\left(s_{0}\right)=1$, which can be embedded in a continuous one parameter group near $s_{0}$, are the linear fractional functions

$$
f(s)=s_{0}+\left(s-s_{0}\right) /\left(1+a\left(s-s_{0}\right)\right) \text {. }
$$

Thus in this case the possibility of embedding is rare.

A fixed point $s_{0}$ for which $f^{\prime}\left(s_{0}\right)=1$ is a multiple root of the equation $f(s)=s$. Thus the result of Baker is concerned with a multiple fixed point. We study a

Received by the editors February 13, 1967.

(1) Research supported in part by contracts NONR 00014-0015 and NSF GP 4784 at Stanford University. 
related problem in which the fixed points are simple (i.e., not multiple) but in which there are two fixed points $s_{0}, s_{1}$ and the segment $\left[s_{0}, s_{1}\right]$ joining them is mapped onto itself by $f(s)$. We ask whether $f(s)$ can be embedded in a continuous one parameter group of functions each regular in a neighborhood of the segment. We prove a result similar to that of Baker but valid for an even wider class of functions than the meromorphic functions. The following definition is needed.

Definition. A function $g(s)$ is of class $\mathscr{S}$ if there is a closed countable set $S$ in the extended complex plane and $g(s)$ is regular and one valued in the complement of $S$.

THEOREM. Let $f(s)$ be a function of class $\mathscr{S}$ with two fixed points $s_{0}, s_{1}$ such that the segment $\left[s_{0}, s_{1}\right]$ is in the domain of regularity of $f(s)$ and is mapped onto itself by $f(s)$. Assume that $0<\left|f^{\prime}\left(s_{0}\right)\right|<1<\left|f^{\prime}\left(s_{1}\right)\right|$ and that for $s$ in the open segment $\left(s_{0}, s_{1}\right)$, $f(s) \neq s, f^{\prime}(s) \neq 0$. Then there is a continuous one parameter group of functions $f(s ; t)$ with cormmon fixed points $s_{0}, s_{1}$ and invariant segment $\left[s_{0}, s_{1}\right]$ such that $f(s ; 1)=f(s)$ if and only if $f(s)$ is a linear fractional function.

Our interest in the embedding problem stems from applications in the theory of Markoff branching processes. Here one has a probability generating function

$$
f(s)=\sum_{k=0}^{\infty} c_{k} s^{k}, \quad c_{k} \geqq 0, \quad \sum c_{k}=1 .
$$

The iterates $f_{n}(s)$ defined by $f_{0}(s)=s$ and

$$
f_{n+1}(s)=f_{n}(f(s)), \quad n=1,2, \ldots
$$

are all probability generating functions and map the unit disc $|s| \leqq 1$ into itself. They satisfy $f_{n}(1)=1$. The related Markov process is called supercritical if $m=f^{\prime}(1)$ $=\sum k c_{k}>1$. In this case, if either $f(0)>0$ or $f(0)=0$ and $f^{\prime}(0)>0$, then there is a unique fixed point $s_{0}$, such that $0 \leqq s_{0}<1$, and we have $f^{\prime}\left(s_{0}\right)=c, 0<c<1$. The question of interest here is whether $f(s)$ can be embedded in a continuous one parameter semigroup of probability generating functions $f(s ; t), 0 \leqq t<\infty$.

If the embedding exists then for every rational $t>0$ we have $f\left(s_{0} ; t\right)=s_{0}$. We prove only the case $f(0)>0$. For any $t>0$ we can choose a positive integer $n$ so that $n t>1$ and hence

$$
f(0 ; n t)=f(f(0 ; 1) ; n t-1)
$$

which is positive since $f(0 ; 1)=f(0)>0$. Also from $f(s ; t+\tau)=f(f(s ; t) ; \tau)$ and continuity follows

$$
f^{\prime}(1 ; t)=m^{t}>1 \text {. }
$$

Therefore for each $t>0$ there is a unique fixed point $s(t), 0<s(t)<1$, and simple calculations show that $s(t)=s(1)=s_{0}$ if $t$ is rational. It then follows from continuity assumptions that $s(t)=s_{0}$ for all $t>0$. 
If the embedding is possible, then the coefficient $c_{1}(t)=f^{\prime}\left(s_{0} ; t\right)$ will be a continuous solution of $c_{1}(t+\tau)=c_{1}(t) c_{1}(\tau)$; hence $c_{1}(t)>0,0 \leqq t<\infty$. Consequently each of the functions will map the segment $\left[s_{0}, 1\right]$ onto itself and $f(s ; t) \neq s$, $s_{0}<s<1$. It is shown in Karlin and McGregor [3] that if we also assume that $f(s)$ is regular at $s=1$, then for each $t \geqq 0 ; f(s ; t)$ is regular at $s=1$. If this be the case, then the functions $f(s ; t)$ have inverses regular on $\left[s_{0}, 1\right]$ which map $\left[s_{0}, 1\right]$ onto itself and hence $f(s)$ is embeddable in a continuous group of analytic functions regular on $\left[s_{0}, 1\right]$ with $\left[s_{0}, 1\right]$ as an invariant segment. The result stated above therefore gives the following conclusion.

THEOREM. If $f(s)$ is a probability generating function of class $\mathscr{S}$, regular at $s=1$ with $f^{\prime}(1)>1$ and either $f(0)>0$ or $f(0)=0$ and $f^{\prime}(0)>0$, then $f(s)$ can be embedded in a continuous one parameter semigroup of probability generating functions if and only if $f(s)$ is a linear fractional function.

In the subcritical case $m=f^{\prime}(1)<1$ it may happen that there is a second fixed point $s_{1}>1$ such that $f(s)$ is regular in some disc $|s|<s_{1}+\varepsilon$. This will always be the case if $f(s)$ is meromorphic. The function $g(s)=f\left(s s_{1}\right) / s_{1}$ is then a probability generating function in the supercritical case with fixed points at $1 / s_{1}$ and at 1 . If $f(s)$ is embedded in a one parameter semigroup $f(s ; t)$ of probability generating functions then so is $g(s)$,

$$
g(s ; t)=f\left(s s_{1} ; t\right) / s_{1},
$$

and the same result applies.

2. The embedding near a simple fixed point. Let $f(s)$ be any analytic function regular at a fixed point $s_{0}$ with $f^{\prime}\left(s_{0}\right)=c, 0<|c|<1$. Then, uniformly in a neighborhood of $s_{0}$ we have

$$
\lim _{n \rightarrow \infty} \frac{f_{n}(s)-s_{0}}{c^{n}}=A(s)
$$

This is well known, but for completeness we outline a method of proof. It will be shown that the convergence of (2.1) is valid in any connected domain $N$ which contains $s_{0}$ and such that $s \in N$ implies $f(s) \in N, f^{\prime}(s) \neq 0, f_{n}(s) \rightarrow s_{0}$ as $n \rightarrow \infty$. Any sufficiently small disc with center $s_{0}$ satisfies these conditions.

By differentiation of $f_{n+1}(s)=f\left(f_{n}(s)\right)$ we obtain

$$
\begin{aligned}
f_{n+1}^{\prime}(s) & =f^{\prime}(s) f^{\prime}(f(s)) f^{\prime}\left(f_{2}(s)\right) \cdots f^{\prime}\left(f_{n}(s)\right), \\
f_{n+1}^{\prime \prime}(s) / f_{n+1}^{\prime}(s) & =f_{n}^{\prime \prime}(s) / f_{n}^{\prime}(s)+\left[f^{\prime \prime}\left(f_{n}(s)\right) / f^{\prime}\left(f_{n}(s)\right)\right] f_{n}^{\prime}(s) .
\end{aligned}
$$

Since $f^{\prime}\left(f_{n}(s)\right) \rightarrow f^{\prime}\left(s_{0}\right)=c$ and $0<|c|<1$ we deduce from (2.2) that $f_{n}^{\prime}(s) \rightarrow 0$ geometrically and $\sum_{n=1}^{\infty} f_{n}^{\prime}(s)$ converges uniformly in any compact part of $N$. Then, from (2.3)

$$
\sum_{n=1}^{\infty}\left[f_{n+1}^{\prime \prime}(s) / f_{n+1}^{\prime}(s)-f_{n}^{\prime \prime}(s) / f_{n}^{\prime}(s)\right]
$$


converges uniformly in any compact part of $N$, and hence likewise for

$$
\lim _{n \rightarrow \infty}\left(f_{n}^{\prime \prime}(s) / f_{n}^{\prime}(s)\right)=\sigma(s)
$$

By integration we find

$$
\lim _{n \rightarrow \infty}\left[f_{n}^{\prime}(s) / c^{n}\right]=\exp \int_{s_{0}}^{s} \sigma(\xi) d \xi
$$

and integrating once more shows that (2.1) converges uniformly in any compact part of $N$.

The limit $A(s)$ is regular at $s_{0}$ and satisfies

$$
A(f(s))=c A(s), \quad A\left(s_{0}\right)=0, \quad A^{\prime}\left(s_{0}\right)=1 .
$$

We solve $w=A(s)$ to find the inverse function $s=B(w)$. It is regular at $w=0$ and satisfies Schröder's functional equation

$$
f(B(w))=B(c w), \quad B(0)=s_{0}, \quad B^{\prime}(0)=1 .
$$

The coefficients in the power series $B(w)=\sum_{0}^{\infty} b_{k} w^{k}$ are uniquely determined by (2.5).

The iterates of $f(s)$, and of the inverse function $f_{-1}(s)$, are expressed by the formula

$$
f_{n}(s)=B\left(c^{n} A(s)\right), \quad n=0, \pm 1, \pm 2, \ldots,
$$

because $B(w)$ is regular in a disc $|w|<\rho$, and for any given $n, c^{n} A(s)$ will lie in this disc for all $s$ in some neighborhood of $s_{0}$. It then follows from (2.4) and (2.5) that, for fixed $n,(2.6)$ is valid in this neighborhood of $s_{0}$.

Let $\alpha$ be the real determination of $\alpha=\log c<0$. Then we obtain an embedding of $f(s)$ in a one-parameter group of functions, with common fixed point $s_{0}$, setting

$$
f(s ; t)=B\left(e^{\alpha t} A(s)\right),
$$

and we obtain other such embeddings by replacing $\alpha$ by

where $\nu$ is an integer,

$$
\alpha_{v}=\alpha+2 \pi i v
$$

$$
f^{(v)}(s ; t)=B\left(e^{\alpha_{v} t} A(s)\right), \quad v=0, \pm 1, \pm 2, \ldots
$$

For any fixed $\nu$ this has an expansion

$$
f^{(v)}(s ; t)=s_{0}+\sum_{k=1}^{\infty} c_{k}(t)\left(s-s_{0}\right)^{k}
$$

where the coefficients $c_{k}(t)$ are continuous functions of $t$. Let us verify that (2.8) gives all embeddings of $f(s)$ with this continuity property. In fact, it follows from $f(s ; t+\tau)=f(f(s ; t) ; \tau)$ that $c_{1}(t)$ must be a solution of

$$
c_{1}(t+\tau)=c_{1}(t) c_{1}(\tau), \quad c_{1}(1)=c
$$

and the only continuous solutions are

$$
c_{1}(t)=\exp t(\alpha+2 \pi i v)
$$


where $v$ is an integer. The remaining coefficients in (2.9) are then uniquely determined by repeated differentiation of $f(f(s) ; t)=f(f(s ; t))$ at $s=s_{0}$. Because of the uniqueness the resulting determination of (2.9) must coincide with (2.8) for the chosen value of $\nu$. Throughout our discussion the term continuous group refers to continuity of the coefficients $c_{k}(t)$ in the expansion about a fixed point.

3. Embedding in a neighborhood of two fixed points. For a function $f(s)$ with two simple fixed points $s_{0}$ and $s_{1}$ we can transform $f$ by the function

to obtain a new function

$$
\varphi(s)=s_{0}+\left(s_{1}-s_{0}\right) s,
$$

$$
g(s)=\left(\varphi_{-1} f \varphi\right)(s)=\frac{f\left(s_{0}+\left(s_{1}-s_{0}\right) s\right)-s_{0}}{s_{1}-s_{0}}
$$

which has simple fixed points at 0 and 1 with $g^{\prime}(0)=f^{\prime}\left(s_{0}\right), g^{\prime}(1)=f^{\prime}\left(s_{1}\right)$. The function $g(s)$, whose iterates $g_{t}$ are the transforms $\varphi_{-1} f_{t} \varphi$ of the iterates of $f$, can be embedded in a continuous one parameter group of functions $g(s ; t)$ each analytic on the segment $[0,1]$ and leaving that segment invariant, if and only if $f(s)$ can be embedded in a continuous one parameter group $f(s ; t)$ with invariant segment $\left[s_{0}, s_{1}\right]$. We note that $f^{\prime}(s)$ is necessarily real valued on the segment.

After this preliminary discussion we now assume that $s_{0}=0, s_{1}=1$ and $f^{\prime}(0)=c$, $f^{\prime}(1)=m$ where $0<c<1<m, f(s) \neq s$ and $f^{\prime}(s) \neq 0$ for $0<s<1$. The inverse function $f_{-1}(s)$ has the same fixed points and $f_{-1}^{\prime}(1)=m^{-1}<1$, so the discussions of $\S 2$ apply. The limit

$$
A_{*}(s)=\lim _{n \rightarrow \infty}\left[f_{-n}(s)-1\right] / m^{-n}
$$

exists uniformly in a neighborhood of $s=1$ and

$$
A_{*}(f(s))=m A_{*}(s), \quad A_{*}(1)=0, \quad A_{*}^{\prime}(1)=1 .
$$

The inverse function $B_{*}(w)$ satisfies

$$
f\left(B_{*}(w)\right)=B_{*}(m w), \quad B_{*}(0)=1, \quad B_{*}^{\prime}(0)=1 .
$$

The most general embedding of $f$ in a continuous one parameter group of functions with common fixed point at $s=1$ is of the form

$$
f_{*}^{(\mu)}(s ; t)=B_{*}\left(e^{t(a+2 \pi i \mu)} A_{*}(s)\right), \quad \mu=0, \pm 1, \pm 2, \ldots
$$

where $a$ is the real determination of $\log m$.

The most general embedding of $f(s)$ in a continuous one parameter group or semigroup with common fixed point $s=0$ is of the form (2.8) where

$$
A(s)=\lim _{n \rightarrow \infty}\left[f_{n}(s) / c^{n}\right]
$$

and $B(w)$ is the inverse function.

Now assume there is an embedding in a continuous one parameter group or 
semigroup such that each function is analytic in some neighborhood of the closed segment $[0,1]$ and maps the segment onto itself. Then clearly near $s=0$ the embedding must coincide with (2.8) for $\nu=0$ and coincide near $s=1$ with (3.3) for $\mu=0$.

Our assumption that $f(s) \neq s$ for $0<s<1$ implies that $f_{n}(s) \rightarrow 0$ as $n \rightarrow+\infty$ for each $s$ in $[0,1)$, and by continuity there is a neighborhood $N_{0}$ of $[0,1)$ in which (3.4) exists. Similarly there is a neighborhood $N_{2}$ of $(0,1]$ in which $(3.1)$ exists. Since $f^{\prime}(s) \neq 0$ on $(0,1)$ we see from $(2.2)$ that $f_{n}^{\prime}(s) \neq 0$ on $(0,1)$, and then by differertiating $A\left(f_{n}(s)\right)=c^{n} A(s)$ and noting that $f_{n}(s) \rightarrow 0$ and $A^{\prime}(0)=1$, we find that $A^{\prime}(s) \neq 0$ on $(0,1)$. Similarly $A_{*}^{\prime}(s) \neq 0$ on $(0,1)$. Evidently $A(s)$ is strictly increasing on $(0,1)$ and we see by letting $s \rightarrow 1$ in $A(f(s))=c A(s)$ that $A(s) \rightarrow+\infty$ as $s \rightarrow 1-$. Similarly $A_{*}(s)$ is strictly monotone on $(0,1)$ and $A_{*}(s) \rightarrow-\infty$ as $s \rightarrow 0+$. Consequently $B(w)$ is regular on $[0, \infty), B_{*}(w)$ is regular on $(-\infty, 0]$, and each member of the equation

$$
f(s ; t)=B\left(e^{\alpha t} A(s)\right)=B_{*}\left(e^{a t} A_{*}(s)\right)
$$

can be continued analytically along the segment $(0,1)$. For each $s$ in $[0,1), B\left(e^{\alpha t} A(s)\right)$ is an analytic function of $t$ regular at $t=0$, that is, for all $t$ in some 0 -neighborhood, $e^{\alpha t} A(s)$ is in the domain of regularity of $B(w)$ and

$$
\left.\frac{\partial f(s ; t)}{\partial t}\right|_{t=0}=\alpha B^{\prime}(A(s)) A(s)=\alpha \frac{A(s)}{A^{\prime}(s)}
$$

The derivative can be computed similarly from the last member of (3.5) and hence

$$
\alpha A(s) / A^{\prime}(s)=a A_{*}(s) / A_{*}^{\prime}(s), \quad 0<s<1 .
$$

From this we have

$$
A(s)=\left[K / A_{*}(s)\right]^{\gamma}, \quad 0<s<1,
$$

where $\gamma=-\alpha / a$ and $K$ is a constant, or equivalently

$$
B_{*}(w)=B\left(\tilde{K} / w^{\gamma}\right), \quad-\infty<w<0,
$$

where $\widetilde{K}$ is a constant. The validity of (3.7) can be extended by analytic continuation to the domain of analytic existence of either member of the equation.

The remainder of the proof is divided into several lemmas.

LEMMA 1. If $f(s)$ is of class $\mathscr{S}$, then $B_{*}(w)$ is also a function of class $\mathscr{S}$.

The proof is based on analytic continuation of $B_{*}(w)$ using the identity

$$
f\left(B_{*}(w)\right)=B_{*}(m w), \quad m=f^{\prime}(1)>1 .
$$

The singular points of $f(s)$ form a closed countable set $S$. In a sufficiently small disc with center at $w=0$ the function $B_{*}(w)$ is regular. Suppose that for some $r>0$ there is a countable set $T_{r}$ in the disc $D_{r}=\{w ;|w|<r\}$ such that $B_{*}(w)$ is regular 
and single valued in $D_{r}-T_{r}$. If $w \in D_{m r}$ then $w / m \in D_{r}$. For each $s \in S$ there are at most countably many points $w \in D_{r}-T_{r}$ such that $B_{*}(w)=s$. Hence, since $S$ is countable, the set

$$
T_{m r}=\left\{w ; w \in D_{m r}, w / m \in T_{r}, \quad \text { or } \quad w / m \in D_{r}-T_{r} \quad \text { and } \quad B_{*}(w / m) \in S\right\}
$$

is countable. If $w_{1} \in D_{m r}-T_{m r}$ then $f\left(B_{*}(w / m)\right)$ is regular at $w=w_{1}$, and hence

$$
B_{*}(w)=f\left(B_{*}(w / m)\right)
$$

defines an analytic continuation of $B_{*}$ to a single valued function regular in $D_{m r}-T_{m r}$. It follows, since $m>1$, that $B_{*}(w)$ is of class $\mathscr{S}$.

LEMMA 2. The singular points of $B_{*}(w)$ form a bounded set, $B_{*}(w)$ vanishes at $\infty$, and $\gamma$ is a positive integer.

Proof. Since $B_{*}(w)$ is of class $\mathscr{S}$, in particular single valued near $\infty$, (3.7) shows, since $B(w)$ is regular at $w=0$ and $B^{\prime}(0)=1$, that $\gamma$ is an integer. Since

$$
\gamma=-(\log c) / \log m>0,
$$

$\gamma$ is a positive integer. Again from (3.7), $B_{*}(w)$ is regular at $\infty$ and vanishes there. It follows that the set of singular points of $B_{*}(w)$ is bounded.

If $g(s)$ is any nonconstant function of class $\mathscr{S}$, its singular points form a closed countable set $S$. This set can be represented as the union $S=S_{0} \cup S_{0}^{\prime}$ where $S_{0}$ is the set of isolated points of $S$ and $S_{0}^{\prime}$ is the set of non-isolated points of $S$. Every point in $S_{0}^{\prime}$ is a limit point of $S_{0}$, otherwise we should have a point $z$ in $S_{0}^{\prime}$ and a closed disc $D$ with center at $z$ such that $D \cap S$ is a nonvoid closed set without isolated points. By the category theorem such a set is uncountable, which is impossible in our case.

Definition. A singular point $z$ of $g(s)$ is called of type 1 if the range of $g(s)$ in every neighborhood of $z$ is dense in the complex plane. For example a pole is not of type 1 but an isolated essential singularity is of type 1 .

Lemma 3. Let $g(s)$ be a nonconstant function of class $\mathscr{S}$ and let $z$ be a nonisolated singular point of $g$. Then $z$ is of type 1 .

Proof. The assertion is clear if $z$ is a limit point of isolated essential singularities of $g(s)$, so it will be sufficient to consider the case when some neighborhood $N$ of $z$ contains no isolated singularities other than poles. We can choose $N$ to be a closed disc with center $z$ since $S$, the set of singularities of $g(s)$, is countable, such that no singular points lie on the boundary of $N$. Suppose, to the contrary, that $z$ is not of type 1 . Then if $N$ is small enough $g(s)$ is bounded away from some finite complex value $b$ in $N$, and $h(s)=[g(s)-b]^{-1}$ is bounded in $N$. Hence $h(s)$ has no isolated singularities in $N$. Since $h(s)$ is regular at every point in $N$ where $g(s)$ is regular, the singularities of $h(s)$ in $N$ form a closed countable set without isolated 
points. By the category argument this set must be void, so $h(s)$ is regular at $z$. But $z$ is a limit point of poles of $g(s)$, so a limit point of zeros of $h(s)$. Hence $h(s)$ is constant and this violates the hypothesis that $g(s)$ is not constant. Thus the singularity of $g(s)$ at $z$ must be of type 1 .

LEMMA 4. $B_{*}(w)$ and $f(s)$ are rational functions.

Proof. First we note that if $B_{*}(w)$ has a singularity of type 1 at some point $w=w_{1}$ then (3.9) shows that the range of $f$ is dense in the complex plane, and then (3.8) shows that $B_{*}(w)$ also has a singularity of type 1 at $w=m w_{1}$. Hence all of the points $w_{1}, m w_{1}, m^{2} w_{1}, \ldots$ are singularities of type 1 for $B_{*}(w)$. Since $m>1$ this violates our earlier conclusion that the singularities of $B_{*}(w)$ form a bounded set. Hence the only singularities of $B_{*}(w)$ are poles, finite in number, and $B_{*}(w)$ vanishes at $\infty$, so is a rational function. Next we note from (3.8) that if $f(s)$ has a singular point of type 1 in the range of $B_{*}(w)$ then $B_{*}(w)$ would also have a singular point of type 1 , which is not the case. Moreover $B_{*}(w)$, like all rational functions, assumes every complex value so that every singular point of $f(s)$ is in the range of $B_{*}(w)$. Consequently every singularity of $f(s)$, including that at $\infty$ if it occurs, is a pole, and $f(s)$ is a rational function.

We can now complete the proof of the main theorem and show that $f(s)$ is a linear fractional function. We have $f(s)=U(s) / V(s)$ where $U(s), V(s)$ are polynomials with no common root. Let $p=\max$ (degree $U$, degree $V$ ). Then $f(s)$ assumes each complex value exactly $p$ times. We assume $p>1$ and obtain a contradiction. We can choose a finite complex value $\zeta$ so that none of the following conditions are satisfied:

(a) $f^{\prime}(\zeta)=0$

(b) $B_{*}(w)=\zeta$ has a solution such that $B_{*}^{\prime}(w)=0$.

Let $n$ be the number of times $B_{*}(w)$ assumes each complex value. Then because of (b), the equation $B_{*}(w)=\zeta$ has $n$ distinct solutions $w_{1}, \ldots, w_{n}$. And because of (a), since $p>1$ there is a value $\xi \neq \zeta$ such that $f(\xi)=f(\zeta)=c$. Let $w_{n+1}$ be a solution of $B_{*}(w)=\xi$. Then the equation $B_{*}(m w)=c$ has the $n+1$ distinct solutions $w_{1}, w_{2}, \ldots$, $w_{n+1}$ which is impossible. This contradiction proves that $p=1$ and $f(s)$ is linear fractional.

4. Further remarks and examples. When the condition that $f(s)$ be single valued under analytic continuation is dropped, one can find embeddable functions in great profusion from the transformation

$$
g=\varphi_{-1} f \varphi
$$

Here we can take for $\varphi$ any function which is regular in a neighborhood of the fixed segment $\left[s_{0}, s_{1}\right]$, maps the segment onto itself, and satisfies $\varphi^{\prime}(s) \neq 0$ on the open segment $\left(s_{0}, s_{1}\right)$. If $\varphi$ has a zero at the end $s=s_{0}$, then the composite $f \varphi$ has there a zero of the same order so that $g=\varphi_{-1} f \varphi$ is regular at $s_{0}$. Similar remarks 
apply to the end $s=s_{1}$. If $f$ is embeddable, in particular if $f$ is linear fractional with iterates $f_{t}$, then $g$ is also embeddable with iterates

$$
g_{t}=\varphi_{-1} f_{t} \varphi
$$

Particularly interesting examples arise from the probability generating function

$$
f(s)=c s /(1-(1-c) s), \quad 0<c<1
$$

where $\left(s_{0}=0, s_{1}=1\right)$ and the function

$$
\varphi(s)=s^{k}, \quad k=2,3, \ldots
$$

In this case we obtain

$$
g(s)=\varphi_{-1}(f(\varphi(s)))=c^{1 / k} s\left[1-(1-c) s^{k}\right]^{1 / k}
$$

which is again a probability generating function.

Transformation of (4.3) by the function

$$
\psi(s)=1-(1-s)^{l}, \quad l=2,3, \ldots
$$

leads to an embeddable function

$$
g(s)=\psi_{-1}(f(\psi(s)))
$$

which is not a probability generating function. Transformation of $f$ by either of the composite functions

$$
\varphi(\psi(s))=\left[1-(1-s)^{l}\right]^{k}, \quad \psi(\varphi(s))=1-\left(1-s^{k}\right)^{l}
$$

leads to further embeddable functions and such transformations can be iterated.

\section{REFERENCES}

1. I. N. Baker, Fractional iteration near a fixed point of multiplier 1, J. Austral. Math. Soc. 4 (1964), 143-148.

2. E. Jabotinsky, Analytic iteration, Trans. Amer. Math. Soc. 108 (1963), 457-477.

3. S. Karlin and J. McGregor, Embeddability of discrete time simple branching processes into continuous time branching processes, Trans. Amer. Math. Soc. 132 (1968), 115-136.

4. G. Szekeres, Regular iteration of real and complex functions, Acta. Math. 100 (1960), 203-258.

STANFORD UNIVERSITY,

Stanford, California 